\title{
Adiponectin diminishes platelet aggregation and sCD40L release. Potential role in the metabolic syndrome
}

\author{
P. Restituto, ${ }^{1}$ I. Colina, ${ }^{2}$ J. J. Varo, ${ }^{3}$ and N. Varo ${ }^{1}$ \\ ${ }^{1}$ Servicio de Bioquímica, ${ }^{2}$ Department of Internal Medicine, and ${ }^{3}$ Family Medicine, Clínica Universidad de Navarra, \\ Pamplona, Spain
}

Submitted 9 December 2009; accepted in final form 24 February 2010

\begin{abstract}
Restituto P, Colina I, Varo JJ, Varo N. Adiponectin diminishes platelet aggregation and $\mathrm{SCD} 40 \mathrm{~L}$ release. Potential role in the metabolic syndrome. Am J Physiol Endocrinol Metab 298: E1072-E1077, 2010. First published March 2, 2010; doi:10.1152/ajpendo.00728.2009.-The proinflammatory and proatherogenic mediator, soluble CD40 ligand (CD40L), is increased in the metabolic syndrome (MS) and released from platelets. We hypothesized that adiponectin modulates platelet function, and we sought to evaluate the association of adiponectin and sCD40L levels with platelet aggregation in MS and the effects of adiponectin on platelet aggregation and activation. Platelet aggregation and circulating adiponectin, sCD40L and P-selectin were determined in 30 controls and 30 patients with MS. Also, in vitro studies were performed in platelet-rich plasma from nine healthy volunteers. Adiponectin receptors were demonstrated by Western blotting and flow cytometry. ADP and epinephrine platelet aggregation was measured after preincubation with adiponectin. sCD40L and P-selectin secretion was measured in the supernatants by ELISA. Patients with MS had higher SCD40L and P-selectin than controls $(5.96 \pm 0.50$ vs. $4.28 \pm 0.41 \mathrm{ng} / \mathrm{ml}, P<0.05$, and $151 \pm 8$ vs. $122 \pm 9 \mathrm{ng} / \mathrm{ml}, P<0.05)$. By contrast, adiponectin was lower in patients with MS than in controls $(5.25 \pm 0.30$ vs. $7.35 \pm 0.34$ $\mu \mathrm{g} / \mathrm{ml}, P<0.001)$. Higher platelet aggregation was found in MS. Adiponectin inversely correlated with P-selectin $(R=-0.35, P=$ $0.009)$, sCD40L $(r=-0.24, P=0.05)$ and epinephrine and collagen induced aggregation $(r=-0.80, P=0.005 ; r=-0.70, P=0.011)$. Platelets express the receptors for adiponectin. Platelet aggregatory response to epinephrine and ADP significantly decreased following preincubation with adiponectin $(96 \pm 4$ vs. $23 \pm 3 \%, P<0.001$, and $102 \pm$ 9 vs. $85 \pm 9 \%, P=0.004)$. Adiponectin prevented platelet sCD40L release $(1.63 \pm 0.15$ vs. $2.04 \pm 0.20 \mathrm{ng} / \mathrm{ml}, P<0.001)$. Enhanced platelet aggregation and activation markers are found in MS associated with low adiponectin concentrations. Novel evidence is provided demonstrating that adiponectin has antithrombotic properties, since it inhibits platelet aggregation and platelet activation.
\end{abstract}

soluble CD40 ligand; platelets; platelet activation; P-selectin

THE METABOLIC MYNDROME (MS) consists of the convergence of a variety of risk factors in the same individual and is characterized by a general proinflammatory and prothrombotic state that interacts synergistically, causing or accelerating the progression of atherosclerosis. There are different definitions to describe this syndrome. Currently, the more accepted definitions are those of the National Cholesterol Education Program - Third Adult Treatment Panel (NCEP ATP III) and the World Health Organization (WHO). In both, obesity, insulin resistance, dyslipidemia, and hypertension are considered to underlie the metabolic syndrome. Prothrombosis is not yet included in any definition of MS, but the International Diabetes Federation (IDF) consensus group identifies a prothrombotic state related to MS and

Address for reprint requests and other correspondence: N. Varo, Servicio de Bioquímica, Clínica Universitaria de Navarra, Avda Pío XII 36, 31008 Pamplona, Spain (e-mail: nvaro@unav.es). advises investigating the predictive power of these extra criteria for cardiovascular risk or diabetes. In fact, several investigations demonstrate the association between a prothrombotic state and diabetes (23), obesity (20), and hypertension (17). Furthermore, recent studies demonstrate that obesity increases platelet reactivity (18). Indeed, there are studies that show that patients with MS have higher platelet count (9), increased platelet reactivity and turnover, and lower antiplatelet response to aspirin than controls $(24,25)$.

CD40 ligand (CD40L) is a proinflammatory mediator expressed with its receptor CD40 in a wide variety of cells. There is in vitro and in vivo evidence of their participation in atherothrombosis. In addition to the cellular form of CD40L, there is a soluble form (sCD40L) secreted by activated platelets, which circulates in plasma. Our group has previously described that patients with MS have higher platelet derived soluble CD40L than controls (15).

Adipose tissue, in excess in patients with MS, secretes, among other cytokines, adiponectin. Reduced adiponectin serum levels correlate with obesity (11), insulin resistance (7), and type 2 diabetes (3). In addition to its metabolic actions, adiponectin has anti-inflammatory and antiatherogenic effects through its receptors (AdipoR1 and AdipoR2) expressed on monocytes, smooth muscle cells, and endothelial cells. Furthermore, there are investigations that confer an antithrombotic role on adiponectin (10) and associate platelet activation with low serum adiponectin (4). The aims of the present work were 1) to compare platelet aggregation and sCD40L levels in patients with MS and evaluate its association with adiponectin concentrations and 2) to study the in vitro effects of adiponectin on platelet aggregation and SCD40L release.

\section{METHODS}

Reagents. Recombinant human adiponectin was purchased from Preprotech. Endotoxin level was less than $0.1 \mathrm{ng} / \mu \mathrm{g}$. Epinephrine, L-adenosine 52-diphosphate, and collagen were purchased from Arkray. The anti-adiponectin antibody was purchased from R\&D Systems.

Study population. The study was performed in 30 healthy individuals (controls) and 30 patients with MS attending the Internal Medicine Department of the Clínica Universidad de Navarra for a general check-up. All participants underwent a complete medical examination. MS was diagnosed according to the National Cholesterol Education Program - Adult Treatment Panel III guidelines, with modification of waist criterion into body mass index (BMI $\geq 30 \mathrm{~kg} / \mathrm{m}^{2}$ ). Exclusion criteria were the presence of impaired renal or liver function, arteritis, connective tissue diseases, chronic inflammatory diseases, coronary artery disease, or stroke. None of the patients had taken aspirin, platelet glycoprotein IIb/IIIa inhibitors, or thienopyridines in the previous 6 mo.

Anthropometric measurements, including weight and height, were obtained using standardized techniques. BMI was calculated using the 
formula weight $(\mathrm{kg}) / \mathrm{height}^{2}(\mathrm{~m})$. Blood pressure was measured on the right arm with a mercury sphygmomanometer with the subjects in a seated position and after a 5-min rest. The average of two measurements, at the beginning and end of the visit, was considered.

In one subgroup of the population, platelet aggregation was measured ( 8 controls and 7 patients with MS). In addition, for the platelet in vitro studies, citrated blood was obtained from nine healthy volunteers who had not taken any medication known to affect platelet function in the previous month.

The local Committee on Human Research approved the study, performed in accordance with the Declaration of Helsinki, and all participants provided written informed consent.

Biochemical analyses. Serum and plasma of patients and controls were collected into Vacutainer tubes. Fasting serum glucose, cholesterol, triglycerides, and high-density lipoprotein cholesterol were measured by standard laboratory techniques.

Serum adiponectin and P-selectin were measured by ELISA (R\&D System, Minneapolis, MN) according to the manufacturer's instructions. The detection limits were 0.003 and $0.010 \mathrm{ng} / \mathrm{ml}$, respectively. sCD40L levels in serum and in platelet supernatants were determined by ELISA (BenderMedSystems, Vienna, Austria). The within-assay coefficient of variation for all assays was $<10 \%$.

Human platelet aggregation studies. Platelet-rich plasma (PRP) was prepared by centrifugation of whole citrated blood at $120 \mathrm{~g}$ for 10 $\min$ at $20^{\circ} \mathrm{C}$. Supernatants were drawn into another tube, and the remaining blood was centrifuged at $1,200 \mathrm{~g}$ for $15 \mathrm{~min}$ to obtain platelet-poor plasma (PPP). Platelet count was performed in the automatic hematology analyzer ABX Pentra 60 (Horiba ABX Diagnostics, France). The PRP was diluted with the PPP corresponding to each patient to obtain a final concentration of $400 \times 10^{6}$ platelets $/ \mathrm{ml}$.

Platelet aggregation was measured in the subgroup of 15 patients by activating platelets through the addition of epinephrine (3.34 $\mu \mathrm{g} / \mathrm{ml}$ ), L-adenosine 52-diphosphate (ADP, $1 \mu \mathrm{g} / \mathrm{ml}$ ), or collagen (5 $\mu \mathrm{g} / \mathrm{ml}$ ) for $5 \mathrm{~min}$ at $37^{\circ} \mathrm{C}$ under constant stirring $(10,000 \mathrm{rpm})$ in an automated platelet aggregation recorder (Aggrecorder II; DIC Kyoto Daiichi Chemical, kyoto, Japan). This aggregometer measures the variation in absorbance caused by platelet aggregation after the addition of an inducer (ADP, collagen, epinephrine, etc.) to the PRP. The data used for diagnosis are the aggregation pattern of the maximum aggregation ratio setting PPP at $0 \%$.

Dose-response curves to adiponectin $(0-40 \mu \mathrm{g} / \mathrm{ml})$ were performed in two healthy volunteers to select an adequate concentration to study the effect of adiponectin on platelet aggregation and SCD40L release. PRP from healthy volunteers $(n=9)$ was preincubated with or without adiponectin (final concentration $25 \mu \mathrm{g} / \mathrm{ml}$; Peprotech) for $40 \mathrm{~min}$ at $37^{\circ} \mathrm{C}$ before the addition of the platelet aggregation inducer. The specificity of the effects was evaluated preincubating adiponectin with an anti-adiponectin antibody $(25 \mu \mathrm{g} / \mathrm{ml})$ for $30 \mathrm{~min}$ at $37^{\circ} \mathrm{C}$. Then, the blocked adiponectin was added, and platelets were stimulated with ADP or epinephrine.

Effect of adiponectin on platelet $s C D 40 L$ secretion. To investigate the effects of adiponectin on platelet sCD40L secretion, PRP from each healthy volunteer $(n=9)$ was pretreated with or without adiponectin $(25 \mu \mathrm{g} / \mathrm{ml})$ for $40 \mathrm{~min}$ at $37^{\circ} \mathrm{C}$. Then, maximal sCD40L release was induced through the incubation with L-adenosine 52diphosphate (ADP, $5.5 \mu \mathrm{g} / \mathrm{ml}$ ) for $30 \mathrm{~min}$ at $37^{\circ} \mathrm{C}$. Aliquots were centrifuged at 3,500 rpm for $7 \mathrm{~min}$. Supernatants were collected, and sCD40L was measured by ELISA. Pellets were resuspended in platelet lysis buffer [20 mmol/l Tris (pH 7.4), $150 \mathrm{mmol} / \mathrm{l} \mathrm{NaCl}, 1 \%$ Triton $\mathrm{X}-100$, and $1 \mathrm{mmol} / \mathrm{l}$ EDTA].

Western blotting. Platelet lysates were used to explore the expression of the receptors of adiponectin, AdipoR1 and AdipoR2, by Western blotting. Protein concentration in platelet lysates was assessed by the Bradford method (Bio-Rad, Hercules, CA). Thirty micrograms of protein was fractionated on $12 \%$ polyacrylamide gels and transferred into nitrocellulose membranes. Membranes were blocked (10\% nonfat dry milk in $0.05 \%$ Tween 20-TBS) and incu- bated with a goat monoclonal antihuman AdipoR1 (1:200; Santa Cruz Biotechnology, Santa Cruz, CA) or AdipoR2 (1:200; Santa Cruz Biotechnology). Bound antibody was detected by peroxidase-conjugated secondary antibody (donkey anti-goat 1:10,000, Santa Cruz Biotechnology) and visualized using the ECL-Plus chemiluminescence detection system (Amersham Biosciences, Piscataway, NJ). We included protein extracts from human heart as a negative control.

Flow cytometry. Human platelets from three different healthy donors were analyzed for surface expression of AdipoR1 and AdipoR2 by staining with fluorescein isothiocyanate (FITC)-coupled antibodies. FITC antibodies were added to the PRP at a final concentration of $5 \mu \mathrm{g} / \mathrm{ml}$ and incubated at room temperature for $30 \mathrm{~min}$. Cells were analysed in a Bekcman Coulter Epics XL flow cytometer. Isotopematched IgG was employed as a control. The results are expressed as the mean intensity of fluorescence (MIF) of adiponectin receptors.

Statistical analysis. Statistical analysis was performed using the Statistical Package for the Social Sciences (SPSS) version 15.0. Normal distribution of samples was assessed by the Shapiro-Wilks test. Differences between study groups were evaluated by the Student's $t$-test for normally or Mann-Whitney U-test for nonnormally distributed variables and $\chi^{2}$ statistic for categorical variables. To investigate differences between experimental conditions, paired Student's $t$-test, Wilcoxon signed rank test, and Kruskal-Wallis test were used. Pearson and Spearman's correlation test assessed univariate correlations. Multivariable linear regression analysis was used to determine whether the association between adiponectin and sCD40L and P-selectin was independent of other confounding factors. For all statistical testing, two-sided probability values were reported, and statistical significance was established at $P<$ 0.05. Data are expressed as means \pm SE.

\section{RESULTS}

In vivo studies. The general characteristics of the study population are presented in Table 1. As expected, patients with MS exhibited significantly $(P<0.05)$ higher BMI, systolic blood pressure (SBP), diastolic blood pressure (DBP), glucose, cholesterol, and triglycerides levels and lower HDL-cholesterol levels than controls.

Patients with MS had significantly higher serum levels of the markers of platelet activation sCD40L and P-selectin than controls $(5.96 \pm 0.50$ vs. $4.28 \pm 0.41 \mathrm{ng} / \mathrm{ml}, P<0.05$, and $151 \pm 8$ vs. $122 \pm 9 \mathrm{ng} / \mathrm{ml}, P<0.05)$. By contrast, adiponectin levels were

Table 1. Demographic and clinical characteristics of the study population

\begin{tabular}{lccr}
\hline \hline \multicolumn{1}{c}{ Variables } & $\begin{array}{c}\text { No Metabolic } \\
\text { Syndrome }\end{array}$ & $\begin{array}{c}\text { Metabolic } \\
\text { Syndrome }\end{array}$ & $P$ Value \\
\hline Age, yr & $44 \pm 2$ & $60 \pm 2$ & $<0.001$ \\
Male, \% & 50 & 73 & 0.033 \\
BMI, kg/m & $27 \pm 2$ & $35 \pm 1$ & 0.011 \\
Systolic arterial pressure, mmHg & $127 \pm 6$ & $150 \pm 4$ & 0.012 \\
Diastolic arterial pressure, mmHg & $72 \pm 5$ & $89 \pm 2$ & 0.001 \\
Statins treatment, \% & 0 & 15 & $<0.001$ \\
Antihypertensive treatment, \% & 0 & 25 & $<0.001$ \\
Glucose, mg/dl & $94 \pm 2$ & $116 \pm 4$ & $<0.001$ \\
Total cholesterol, mg/dl & $192 \pm 7$ & $217 \pm 8$ & 0.033 \\
Triglycerides, mg/dl & $76 \pm 6$ & $143 \pm 8$ & $<0.001$ \\
HDL-cholesterol, mg/dl & $60 \pm 3$ & $47 \pm 2$ & $<0.001$ \\
LDL-cholesterol, mg/dl & $121 \pm 7$ & $139 \pm 6$ & $\mathrm{NS}$ \\
Adiponectin, $\mu \mathrm{g} / \mathrm{ml}$ & $7.35 \pm 0.3$ & $5.25 \pm 0.3$ & $<0.001$ \\
sCD40L, ng/ml & $4.28 \pm 0.4$ & $5.96 \pm 0.5$ & 0.013 \\
P-selectin, ng/ml & $122 \pm 9$ & $151 \pm 8$ & 0.025 \\
\hline
\end{tabular}

Values are means $\pm \mathrm{SE} ; n=30$. sCD40L, soluble CD40 ligand; NS, nonsignificant. 
significantly lower in patients with MS than in controls $(5.25 \pm$ 0.30 vs. $7.35 \pm 0.34 \mu \mathrm{g} / \mathrm{ml}, P<0.001$ ) (Fig. 1).

Platelet aggregation induced by ADP or collagen was significantly higher in patients with MS than in the control population (96 \pm 4 vs. $86 \pm 2 \%, P=0.038$, and $99 \pm 3$ vs. $91 \pm 3 \%, P=$ 0.041). Although epinephrine-induced aggregation tended to be higher in patients with MS (98 \pm 3 vs. $89 \pm 3 \%, P=0.08)$, it did not reach statistical significance.

We next explored the association between circulating levels of adiponectin and SCD40L with other biochemical and demographic parameters. sCD40L levels correlated with some factors of MS (triglycerides $r=0.49, P=0.006$, and HDLcholesterol $r=-0.53, P=0.023$; Table 2). Similarly, adiponectin inversely correlated with triglycerides $(r=-0.49, P$ $<0.001)$, BMI $(r=-0.33, P=0.050)$, and glucose $(r=$ $-0.46, P<0.001)$ and directly with HDL-cholesterol $(r=$ $0.59, P<0.001)$. P-selectin and sCD40L concentrations correlated with platelet count $(r=0.44, P=0.006$, and $r=0.35$, $P=0.041$, respectively). Interestingly, in the whole population, levels of adiponectin inversely and significantly correlated with the markers of platelet activation P-selectin $(r=$ $-0.35, P=0.009)$ and sCD40L $(r=-0.24, P=0.05)$ (Fig. $2)$. After adjustments for age and sex, adiponectin significantly associated with $\mathrm{P}$-selectin $(\beta=-0.40, P=0.01)$ and SCD40L ( $\beta=-0.31, P=0.05)$. Importantly, in the subgroup, adiponectin significantly and inversely correlated with epinephrine- and collagen-induced aggregation $(r=-0.80, P=$ 0.005 , and $r=-0.70, P=0.011$, respectively) and nearly

\section{A}

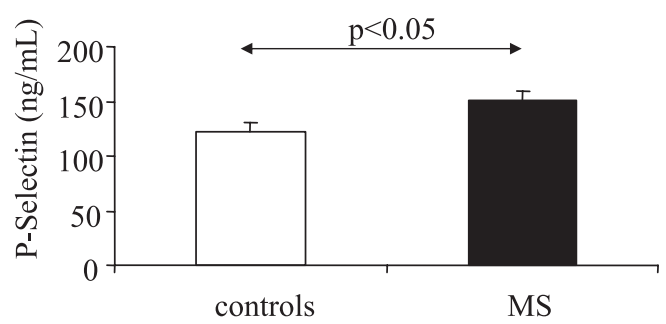

B

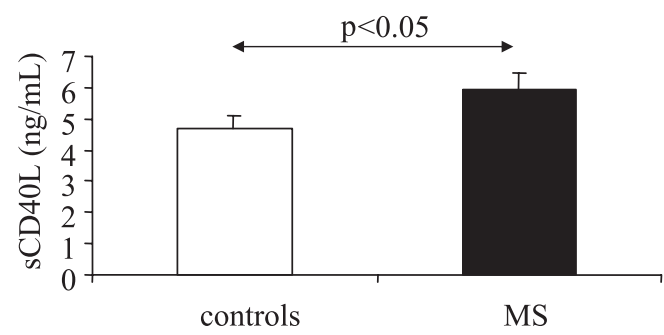

C

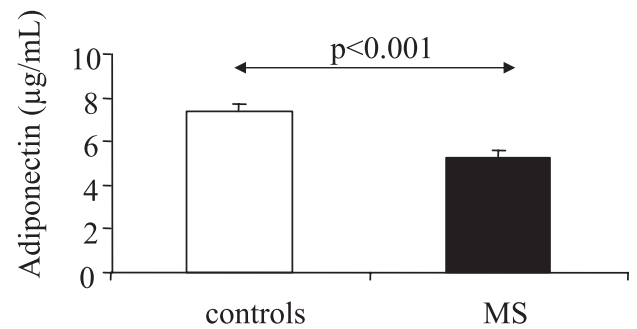

Fig. 1. Serum levels of soluble CD40 ligand (sCD40L), adiponectin, and P-selectin in healthy donors and patients with metabolic syndrome (MS). P-selectin $(A)$, sCD40L $(B)$, and adiponectin $(C)$ serum concentrations were measured by ELISA in patients with and without MS ( $n=30$ in both groups). Histograms represent means $\pm \mathrm{SE}$.
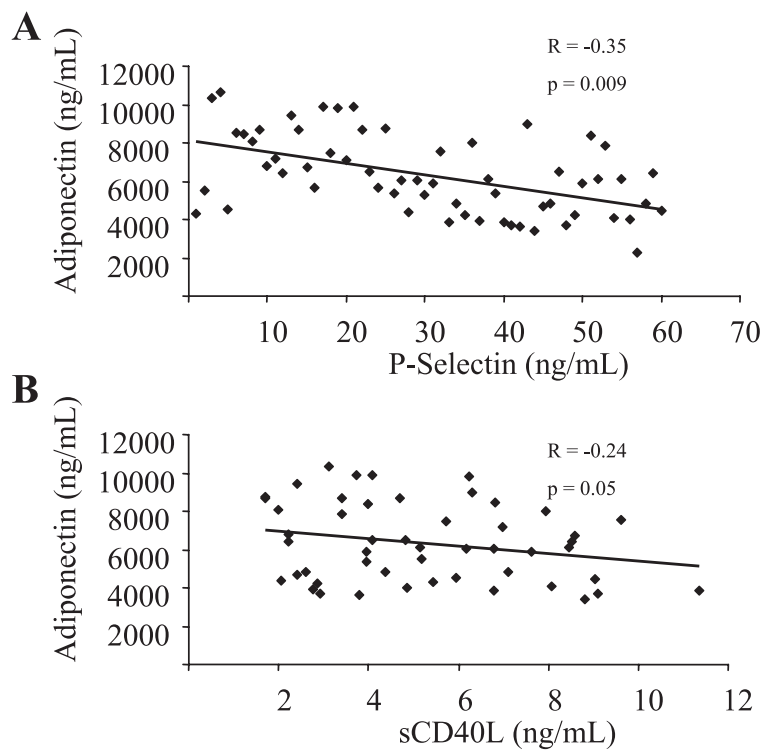

Fig. 2. Correlations of circulating adiponectin with P-selectin and sCD40L. Serum $\mathrm{P}$-selectin, sCD40L, and adiponectin were measured in patients with and without MS (both $n=30$ ). Scatter plots show correlations of circulating adiponectin with $\mathrm{P}$-selectin $(A)$ and SCD40L $(B)$ in patients with and without MS.

reached significance with ADP-induced aggregation $(r=$ $-0.59, P=0.06)$.

Effect of adiponectin on platelet aggregation. To investigate whether platelets could respond to adiponectin, we tested by Western blotting whether platelets from healthy volunteers expressed the receptors for adiponectin. Western blot analysis revealed the presence of immunoreactive bands at the expected molecular mass (AdipoR1, $42 \mathrm{kDa}$; AdipoR2, $35 \mathrm{kDa}$ ) in all donors (Fig. 3). Nonactivated human platelets were analysed by flow cytometry for the expression of adiponectin receptors on the cell surface. An increase in MIF was found for both AdipoR1 and AdipoR2 $(152 \pm 20$ and $539 \pm 22)$ compared with the isotope control. AdipoR1 and AdipoR2 expression was compared in platelets obtained from patients with and without MS. No statistically significant differences were found in AdipoR1 or AdipoR2 in patients with MS compared with controls $(1.52 \pm 0.43$ vs. $1.34 \pm 0.12$ arbitrary units or $2.29 \pm$ 0.87 vs. $1.11 \pm 0.29$ arbitrary units).

Once it was demonstrated that platelets express receptors for adiponectin, we next studied the effects of adiponectin on platelet aggregation by pretreating platelets with increasing concentrations of adiponectin (range $0-40 \mu \mathrm{g} / \mathrm{ml}$ ). Adiponectin dose-depen-
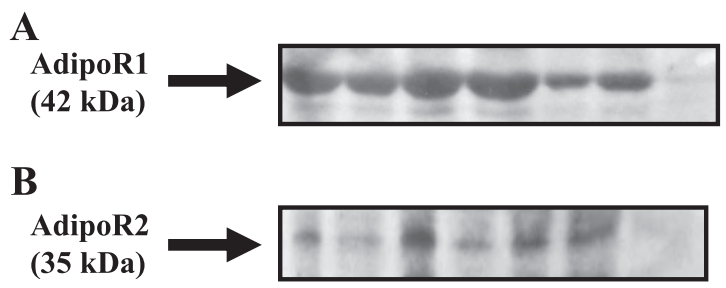

Fig. 3. Platelets express the adipokine receptors AdipoR1 and AdipoR2. Platelet protein extracts $(30 \mu \mathrm{g})$ from 5 healthy donors were separated by SDS-PAGE, transferred to nitrocellulose, and subjected to Western blotting analysis, showing a positive band at the expected molecular weight of AdipoR1 $(A)$ and AdipoR2 $(B)$. The last lane corresponds to a protein extract obtained from human heart employed as a negative control. 
A

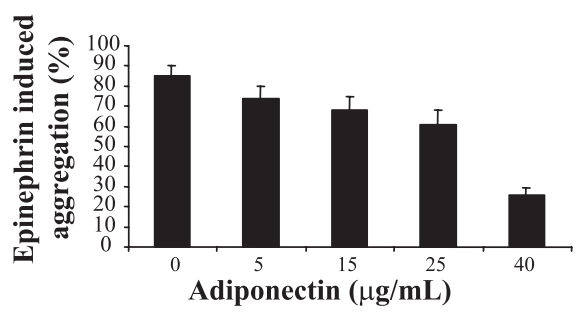

C

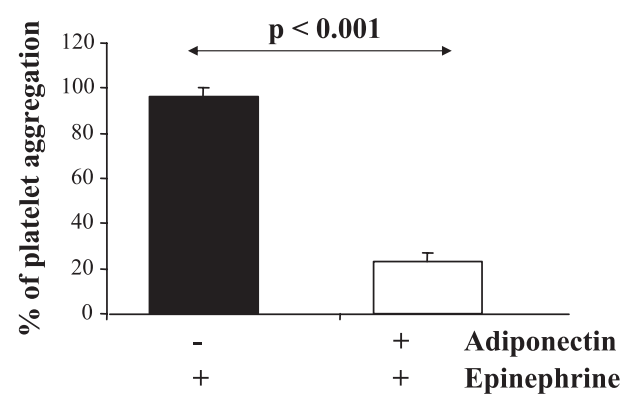

B
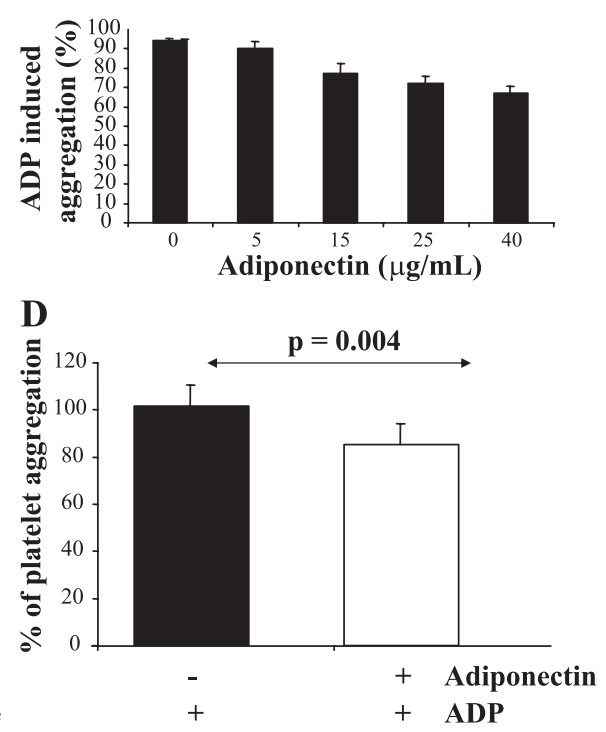

Fig. 4. Adiponectin diminishes platelet aggregation. Platelet-rich plasma $(n=2)$ was incubated with increasing concentrations of adiponectin $(0-40 \mu \mathrm{g} / \mathrm{ml})$. Light transmission aggregometry was used to assess degree of platelet aggregation in response to epinephrine $(A)$ and $\operatorname{ADP}(B)$. Epinephrine $(C)$ and $\operatorname{ADP}$ $(D)$ aggregation was measured in healthy donors $(n=9)$ after incubation with adiponectin (25 $\mu \mathrm{g} / \mathrm{ml}$ ) for $40 \mathrm{~min}$ at $37^{\circ} \mathrm{C}$. Data are expressed as means $\pm \mathrm{SE}$. dently decreased epinephrine- and ADP-induced aggregation (Fig. 4, $A$ and $B$ ). The effects of adiponectin on collagen-induced aggregation were not so clear, so in follow-up we decided to employ epinephrine and ADP for the in vitro studies. The aggregatory response to epinephrine significantly decreased in platelets following preincubation with adiponectin $(25 \mu \mathrm{g} / \mathrm{ml} ; 96 \pm 4 \mathrm{vs}$. $23 \pm 3 \%, P<0.001$; Fig. $4 C)$. Similarly, adiponectin $(25 \mu \mathrm{g} / \mathrm{ml})$ significantly decreased ADP-induced aggregation (102 \pm 9 vs. $85 \pm 9 \%, P=0.004$; Fig. $4 D$ ).

To ensure that the observed effects were specific to adiponectin, this adipocytokine was preincubated with an antiadiponectin antibody (100 ng/ml, $\left.30 \mathrm{~min}, 37^{\circ} \mathrm{C}\right)$. Subsequently, the blocked adiponectin was added to the platelets $(n=3)$, and they were stimulated with epinephrine or ADP. When adiponectin was preblocked with a specific antibody, there was a reduction of the effect of adiponectin, as epinephrine-induced aggregation of control platelets was $89 \pm 1 \%$, incubated with adiponectin $18 \pm 1 \%$, and incubated with anti-adiponectin antibody $41 \pm 1 \%$. ADP-induced aggregation of control platelets was $88 \pm 1 \%$, with adiponectin $20 \pm 1 \%$, and with the blocked adiponectin $57 \pm 1 \%$. This is a reduction of the effect mediated by adiponectin in epinephrine- and ADP-induced platelet aggregation of 67 and 51\%, respectively.

Effect of adiponectin on P-selectin and $s C D 40 L$ release. We next aimed to study platelet activation by measuring biomarkers released from platelet $\alpha$-granules upon activation with different agonists. ADP was employed as a potent inductor of $\mathrm{SCD} 40 \mathrm{~L}$ and P-selectin release. SCD40L and P-selectin were quantified in platelet supernatants following stimulation and centrifugation. Interestingly, adiponectin significantly and dose-dependently decreased sCD40L release (Fig. 5A). Maximal effect was observed at $25 \mu \mathrm{g} / \mathrm{ml}$. This concentration was selected in order to evaluate the effect in all donors. ADP-induced P-selectin release was significantly prevented by adiponectin (1.77 vs. $1.07 \mathrm{ng} / \mathrm{ml}$ ). ADP significantly increased sCD40L release (nonstimulated vs. stimulated: $1.63 \pm 0.15$ vs. $4.38 \pm 0.31 \mathrm{ng} / \mathrm{ml}, P<0.001$; Fig. $5 B$ ) and preincubation of platelets with adiponectin significantly prevented sCD40L release $(4.38 \pm 0.31$ and $2.04 \pm 0.20 \mathrm{ng} / \mathrm{ml}, P<0.001$;
Fig. 5B). Preincubation of adiponectin with an anti-adiponectin antibody before its addition to the platelets resulted in a $55 \%$ reduction in $\mathrm{SCD} 40 \mathrm{~L}$ platelet release. Adiponectin alone had no effect on $\mathrm{sCD} 40 \mathrm{~L}$ release.

\section{DISCUSION}

The main findings of the current study are the following: 1) $\mathrm{sCD} 40 \mathrm{~L}, \mathrm{P}$-selectin, and platelet aggregation are increased in

$\mathbf{A}$

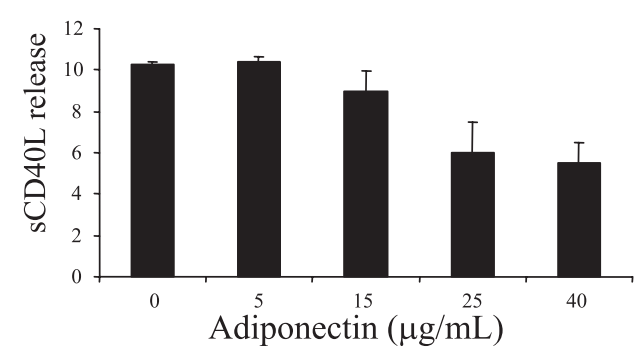

B

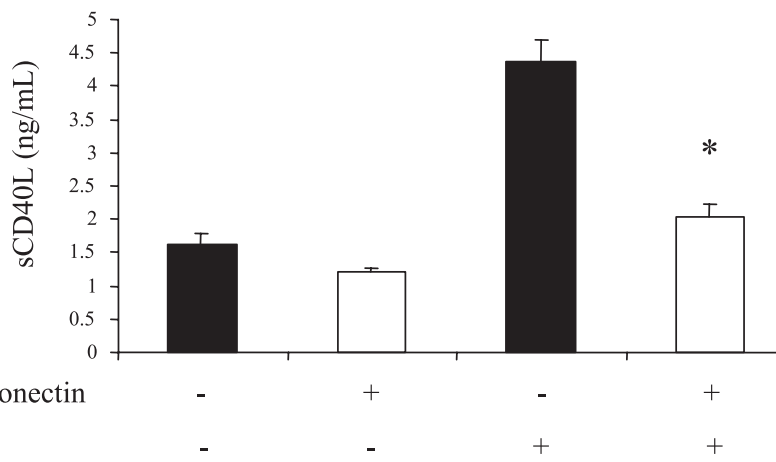

Fig. 5. Adiponectin reduces platelet sCD40L release. Platelet-rich plasma was incubated with increasing concentrations of adiponectin $(0-40 \mu \mathrm{g} / \mathrm{ml})$. Following incubation, sCD40L release was induced with $\mathrm{ADP}\left(5.5 \mu \mathrm{g} / \mathrm{ml}, 30 \mathrm{~min}, 37^{\circ} \mathrm{C}\right)$. Samples were centrifuged $(3,500 \mathrm{rpm}, 7 \mathrm{~min})$ and $\mathrm{SCD} 40 \mathrm{~L}(A)$ was measured in the supernatants by ELISA. Platelet-rich plasma from healthy volunteers was treated with or without adiponectin $\left(25 \mu \mathrm{g} / \mathrm{ml}, 40 \mathrm{~min}, 37^{\circ} \mathrm{C}\right)$, and sCD $40 \mathrm{~L}$ release was measured in the supernatants $(B)$. Histograms represent means $\pm \mathrm{SE}$. $* P<$ 0.05 vs. stimulation with ADP without adiponectin. 
MS and adiponectin inversely correlates with SCD40L, P-selectin, and platelet aggregation; 2) platelets express the adiponectin receptors AdipoR1 and AdipoR2; and 3) adiponectin inhibits platelet aggregation and platelet $\mathrm{P}$-selectin and $\mathrm{sCD} 40 \mathrm{~L}$ release.

Metabolic syndrome and prothrombosis. It is known that patients with MS present a proinflammatory and prothrombotic state. The individual risk factors obesity (2), diabetes (24), and hypertension (29) and also MS (24) have been associated with high platelet activity. The data from the present study show that patients with MS exhibited higher platelet activity compared with control subjects, which is in agreement with previous publications (24). Also, the present study confirms previous reports describing higher levels of the markers of platelet activation P-selectin and sCD40L and lower levels of adiponectin in patients with MS than in controls $(12,14,15,21,22)$. Our group $(15,27)$ previously demonstrated that platelets from patients with diabetes or MS release higher amounts of sCD40L than platelets from controls, which could account for the elevated SCD40L levels observed in these patients. Glucose and advanced glycation end products have been described as potential triggers (26), but other mediators can be involved. Here, we identify adiponectin as a mediator of obesity-related cardiovascular complications that also modulates platelet sCD40L release. As platelets are the main source of circulating sCD40L, it is tempting to speculate that, in conditions where there is a decrease of adiponectin, such as obesity, diabetes, or insulin resistance, the control of platelet activation and aggregation will be diminished. The finding of low adiponectin levels and high aggregation in patients with MS and the negative correlation of adiponectin with $\mathrm{sCD} 40 \mathrm{~L}$ circulating levels supports this hypothesis.

Some studies have evaluated the association of platelet count with MS with controversial results $(9,13,28)$. We did not find differences in platelet count between controls and patients with MS, but sCD40L levels increased along with platelet count.

Effects of adiponectin on platelet aggregation. Adipose tissue is an endodrine organ that releases bioactive molecules known as adipokines. One of them, adiponectin, has been described to present antiatherogenic and anti-inflammatory properties beyond its insulin-sensitizing effects (8). In addition, a proaggregatory platelet phenotype has been described in adiponectin-deficient mice, and recombinant adiponectin inhibited enhanced platelet aggregation (10), providing a new link between adipose tissue and thrombosis. Platelets contribute critically to the development and progression of cardiovascular diseases, but so far there is no evidence of the presence of adiponectin receptors on platelets. The present study demonstrates by two different techniques that this cell type expresses these receptors; thus, platelets comprise a target for new antiatherosclerotic actions of adiponectin. However, very few studies have evaluated the direct effect of adiponectin on platelets. A previous report demonstrated that adiponectin has effects on human and murine platelets (19). The kind of recombinant adiponectin employed is of relevance, as this study showed that recombinant globular adiponectin, but not full-length adiponectin, stimulated platelet aggregation and dense granule secretion. Another study found that low concentrations of adiponectin $(<0.5 \mu \mathrm{g} / \mathrm{ml})$ did not influence the effects of ADP and collagen on platelet adhesion (5).

By contrast, our results show that exposure of platelets to full-length adiponectin at the concentrations that are found in plasma in lean individuals $(10-30 \mu \mathrm{g} / \mathrm{ml})$ inhibited platelet ag- gregation. Also, adiponectin exists in different multimeric isoforms that may have different biological activities, and the highmolecular-weight oligomers correlate better with metabolic parameters (6) (16). Future studies will need to clarify the effect of the different multimeric isoforms on platelet function, but our findings provide a new link between obesity and cardiovascular pathologies and suggest that alteration of adiponectin concentrations could alter the human thromboembolic potential.

Effects of adiponectin on SCD4OL release. Activated platelets express CD40L in the surface that is cleaved and released. The binding of sCD40L to its receptor CD40 participates in the initiation, progression, and weakening of the atheroma plaques. Also, the involvement of the CD40-CD40L pathway in atherothrombosis has been extensively studied (1), although the exact mechanisms underlying this association remain under constant study. We (15) have previously shown that adiponectin downregulates the CD40-CD40L dyad in macrophages and endothelial cells, but so far the effect on platelets has not been investigated. The novel observation that adiponectin is capable of preventing platelet SCD40L release demonstrates that this adipokine has a protector role from platelet activity and provides a new link between obesity and thrombosis.

Limitations. One limitation of the present study is that in vitro platelet aggregation studies have been performed in healthy donors, adding adiponectin to the PRP. Therefore the adiponectin is additional to that already present in the plasma, so these are relatively high concentrations of adiponectin. Although we did not find differences in AdipoR1 and AdipoR2 expression in patients with and without MS, future studies will be required to evaluate whether platelets from different donors respond differently to adiponectin. The finding of adiponectin receptors is preliminary, and more studies should definitively clarify the expression; also, the possibility that adiponectin exerts its effects on platelets by binding to other receptor cannot be excluded. Also, to show specificity of the adiponectin effects, we blocked the adipokine with an antibody before adding it to the platelets. It is possible that the antibody does not bind completely to the portion that interacts

Table 2. Correlations of P-selectin, sCD4OL, and adiponectin in the entire population

\begin{tabular}{lccc}
\hline \hline \multicolumn{1}{c}{ Variables } & \multicolumn{2}{c}{ Correlations } \\
\cline { 2 - 4 } & $R$ & & $P$ \\
\hline & & P-selectin \\
Diastolic blood pressure & 0.36 & & 0.048 \\
Triglycerides, (mg/dl) & 0.54 & & $<0.001$ \\
Cholesterol, (mg/dl) & 0.33 & & 0.038 \\
sCD40L, (ng/ml) & 0.38 & & 0.039 \\
Platelet count (10E9/l) & 0.44 & & 0.006 \\
& & sCD40L & \\
Waist circumference & & & 0.044 \\
HDL <40/50 & -0.51 & & 0.023 \\
Triglycerides & 0.53 & & 0.006 \\
Platelet count & 0.49 & & 0.041 \\
& 0.35 & & \\
BMI & & Adiponectin \\
Triglycerides & -0.33 & & 0.050 \\
HDL-cholesterol & -0.49 & & $<0.001$ \\
Glucose & 0.59 & & $<0.001$ \\
& -0.46 & & $<0.001$ \\
\hline
\end{tabular}


with its receptor, which could explain the reduction in the observed effects, but not the full blockade.

Another limitation is that we employed PRP, not isolated platelets, for the in vitro experiments. According to our experience in previous experiments, washed platelets and PRP yield comparable results when one is evaluating aggregation and release of activation markers. Thus, since obtaining washed platelets is tedious, and very often they activate in the process of isolation, we decided to employ PRP.

In summary, this study describes increased sCD40L and platelet aggregation associated with low adiponectin in MS and provides novel evidence demonstrating that adiponectin has antithrombotic properties, since it inhibits platelet aggregation and activation. These findings suggest that the hypoadiponectinemia found in patients with MS could in part contribute to the increased risk of vascular thrombosis as it indirectly affects hemostasis.

\section{ACKNOWLEDGMENTS}

We acknowledge Nerea Zabalza for skillful assistance in this project.

\section{GRANTS}

This work was supported by Grants 35/2005 from the Department of Health of the Government of Navarre, the Department of Education of the Government of Navarre, and SAF2005-05919 from the Ministry of Science and Education.

\section{DISCLOSURES}

No conflicts of interest are reported by the author(s).

\section{REFERENCES}

1. Antoniades C, Bakogiannis C, Tousoulis D, Antonopoulos AS, Stefanadis C. The CD40/CD40 ligand system: linking inflammation with atherothrombosis. J Am Coll Cardiol 54: 669-677, 2009.

2. Desideri G, De Simone M, Iughetti L, Rosato T, Iezzi ML, Marinucci MC, Cofini V, Croce G, Passacquale G, Necozione S, Ferri C. Early activation of vascular endothelial cells and platelets in obese children. $J$ Clin Endocrinol Metab 90: 3145-3152, 2005.

3. Dibello JR, Baylin A, Viali S, Tuitele J, Bausserman L, McGarvey ST. Adiponectin and type 2 diabetes in Samoan adults. Am J Hum Biol 21: 389-391, 2008.

4. Ekmekçi H, Ekmekçi OB, Erdine S, Sönmez H, Ataev Y, Oztürk Z, Bütün II, Gürel C, Kucur M, Turfaner N, Ulutin T, Purisa S, Vural VA. Effects of serum homocysteine and adiponectin levels on platelet aggregation in untreated patients with essential hypertension. J Thromb Thrombolysis 28: 418-424, 2008.

5. Elbatarny HS, Netherton SJ, Ovens JD, Ferguson AV, Maurice DH. Adiponectin, ghrelin, and leptin differentially influence human platelet and human vascular endothelial cell functions: implication in obesity-associated cardiovascular diseases. Eur J Pharmacol 558: 7-13, 2007.

6. Fujimatsu D, Kotooka N, Inoue T, Nishiyama M, Node K. Association between high molecular weight adiponectin levels and metabolic parameters. J Atheroscler Thromb 16: 553-559, 2009.

7. Guo LL, Pan Y, Jin HM. Adiponectin is positively associated with insulin resistance in subjects with type 2 diabetic nephropathy and effects of angiotensin II type 1 receptor blocker losartan. Nephrol Dial Transplant 24: 1876-1883, 2009.

8. Han SH, Sakuma I, Shin EK, Koh KK. Antiatherosclerotic and antiinsulin resistance effects of adiponectin: basic and clinical studies. Prog Cardiovasc Dis 52: 126-140, 2009.

9. Jesri A, Okonofua EC, Egan BM. Platelet and white blood cell counts are elevated in patients with the metabolic syndrome. J Clin Hypertens (Greenwich) 7: 705-713, 2005.

10. Kato H, Kashiwagi H, Shiraga M, Tadokoro S, Kamae T, Ujiie H, Honda S, Miyata S, Ijiri Y, Yamamoto J, Maeda N, Funahashi T, Kurata Y, Shimomura I, Tomiyama Y, Kanakura Y. Adiponectin acts as an endogenous antithrombotic factor. Arterioscler Thromb Vasc Biol 26: $224-230,2006$
11. Kawano J, Arora R. The role of adiponectin in obesity, diabetes, and cardiovascular disease. J Cardiometab Syndr 4: 44-49, 2009.

12. Lee WL, Lee WJ, Chen YT, Liu TJ, Liang KW, Ting CT, Huey-Herng Sheu W. The presence of metabolic syndrome is independently associated with elevated serum CD40 ligand and disease severity in patients with symptomatic coronary artery disease. Metabolism 55: 1029-1034, 2006.

13. Lohsoonthorn V, Jiamjarasrungsi W, Williams MA. Association of hematological parameters with clustered components of metabolic syndrome among professional and office workers in Bangkok, Thailand. Diabetes Metab Syndr 1: 143-149, 2007.

14. Nam JS, Park JS, Cho MH, Jee SH, Lee HS, Ahn CW, Lowe WL Jr, Kim KR. The association between pulse wave velocity and metabolic syndrome and adiponectin in patients with impaired fasting glucose: cardiovascular risks and adiponectin in IFG. Diabetes Res Clin Pract 84: 145-151, 2009.

15. Natal C, Restituto P, Inigo C, Colina I, Diez J, Varo N. The proinflammatory mediator CD40 ligand is increased in the metabolic syndrome and modulated by adiponectin. J Clin Endocrinol Metab 93: 2319-2327, 2008.

16. Pajvani UB, Du X, Combs TP, Berg AH, Rajala MW, Schulthess T, Engel J, Brownlee M, Scherer PE. Structure-function studies of the adipocyte-secreted hormone Acrp30/adiponectin. Implications for metabolic regulation and bioactivity. J Biol Chem 278: 9073-9085, 2003.

17. Pastor-Perez F, Marin F. Hypertension, aortic sclerosis and the prothrombotic state: understanding the complex interaction. J Hum Hypertens 23: $287-288,2009$

18. Raffaelli F, Nanetti L, D'Angelo M, Montecchiani G, Alidori A, Montesi L, Faloia E, Vignini A, Mazzanti L. Interactions between lipoproteins and platelet membranes in obesity. Obesity (Silver Spring) 17: $1375-1380,2009$

19. Riba R, Patel B, Aburima A, Naseem KM. Globular adiponectin increases cGMP formation in blood platelets independently of nitric oxide. J Thromb Haemost 6: 2121-2131, 2008.

20. Rosito GA, D'Agostino RB, Massaro J, Lipinska I, Mittleman MA, Sutherland P, Wilson PW, Levy D, Muller JE, Tofler GH. Association between obesity and a prothrombotic state: the Framingham Offspring Study. Thromb Haemost 91: 683-689, 2004.

21. Saely CH, Risch L, Hoefle G, Rein P, Muendlein A, Marte T, Aczel S, Langer P, Drexel H. Low serum adiponectin is independently associated with both the metabolic syndrome and angiographically determined coronary atherosclerosis. Clin Chim Acta 383: 97-102, 2007.

22. Salas-Salvado J, Granada M, Bullo M, Corominas A, Casas P, Foz M. Plasma adiponectin distribution in a Mediterranean population and its association with cardiovascular risk factors and metabolic syndrome. Metabolism 56: 1486-1492, 2007.

23. Schneider DJ, Hardison RM, Lopes N, Sobel BE, Brooks MM. Association between increased platelet P-selectin expression and obesity in patients with type 2 diabetes: a BARI 2D (Bypass Angioplasty Revascularization Investigation 2 Diabetes) substudy. Diabetes Care 32: 944-949, 2009.

24. Serebruany VL, Malinin A, Ong S, Atar D. Patients with metabolic syndrome exhibit higher platelet activity than those with conventional risk factors for vascular disease. J Thromb Thrombolysis 25: 207-213, 2008.

25. Vaduganathan M, Alviar CL, Arikan ME, Tellez A, Guthikonda S, DeLao T, Granada JF, Kleiman NS, Ballantyne CM, Lev EI. Platelet reactivity and response to aspirin in subjects with the metabolic syndrome. Am Heart J 156: e1001-1002, e1007, 2008.

26. Varo N, Libby P, Nuzzo R, Italiano J, Doria A, Schonbeck U. Elevated release of sCD40L from platelets of diabetic patients by thrombin, glucose and advanced glycation end products. Diab Vasc Dis Res 2: 81-87, 2005.

27. Varo N, Vicent D, Libby P, Nuzzo R, Calle-Pascual AL, Bernal MR, Fernandez-Cruz A, Veves A, Jarolim P, Varo JJ, Goldfine A, Horton E, Schonbeck U. Elevated plasma levels of the atherogenic mediator soluble CD40 ligand in diabetic patients: a novel target of thiazolidinediones. Circulation 107: 2664-2669, 2003

28. Wang YY, Lin SY, Liu PH, Cheung BM, Lai WA. Association between hematological parameters and metabolic syndrome components in a Chinese population. J Diabetes Complications 18: 322-327, 2004.

29. Winther K, Gleerup G, Hedner T. Enhanced risk of thromboembolic disease in hypertension from platelet hyperfunction and decreased fibrinolytic activity: has antihypertensive therapy any influence? J Cardiovasc Pharmacol 19, Suppl 3: S21-S24, 1992. 